\title{
Characterization of Magnetoliposomes by Analytical Electron Microscopy
}

\author{
Shu-You Li*, Ying Guo*, M. Aslam, Lei Fu and Vinayak P. Dravid \\ *Electron Probe Instrumentation Center (EPIC) of the NUANCE User Facilities, \\ Department of Materials Science \& Engineering, Northwestern University, Evanston, IL 60208
}

Magnetic particles encapsulated inside liposomes (magnetoliposome, ML) are considered as ideal carriers for drug delivery and targeting [1,2] because liposome consists of phospholipids which are components of the natural cell membrane and provide optimal biocompatibility in view of in vivo application. The size and structure of MLs are generally characterized by atomic force microscopy (AFM), transmission electron microscopy (TEM), and static magnetic birefringence (SMB). Among these techniques TEM is believed to be the most convincing approach [3]. However, conventional TEM is not capable of recognizing elemental information which would become more critical as nanoparticles become more chemically complex. To fully characterize the ML core-shell structure nanoscale spectroscopy attached to normal TEM is necessary.

The magnetoliposomes were synthesized with magnetic fluid containing $\mathrm{Fe}_{3} \mathrm{O}_{4}$ nanoparticles, which were prepared by mixture of $\mathrm{FeCl}_{2}$ and $\mathrm{FeCl}_{3}$ solution with addition of ammonium hydroxide[4], and egg-yolk phospholipids (PC, Sigma). Cholysterol (Sigma) was used to stabilize liposome bilayers. First cholysterol was dissolved in vegetable oil and PC was dissolved in ethanol. The two solutions were then mixed together (molar ratio 1:1) and magnetite nanoparticles were added to the mixture. While intensively stirring this mixture, large amount of deionized water $(>90 \%)$ was added to make milky suspension. This suspension separated into two fractions after being kept at room temperature for 2-3 days. Magnetoliposomes were obtained in lower part of the solution. The solution is stable for at least 2 months.

Negative stained TEM samples were prepared using uranyl acetate. Figure 1a is a TEM micrograph of the magnetoliposomes with medium magnification. Several MLs were clearly seen in the photograph (indicated by arrows) with existence of pure empty liposomes. Figure $1 \mathrm{~b}$ shows an enlargement of one ML in Fig. 1a. A series of energy dispersive X-ray (EDX) spectrum was obtained from outside ML, edge of ML and center of ML (fig. 1c, 1d and 1e, respectively). The size of the electron beam used in this experiment is about $2 \mathrm{~nm}$ using Hitachi cold field emission gun TEM (HF2000). This spectroscopic data clearly shows the magnetite-phospholipid core-shell structure of ML. In the spectrum from the edge of ML (fig. 1d) we can see the peaks of both iron from $\mathrm{Fe} 3 \mathrm{O} 4$ core and phosphor from phospolipid. Oxygen peak in this region is also higher due to oxygen atoms in phospholipid and/or cholysterol.

From fig $1 \mathrm{a}$ and $1 \mathrm{~b}$, the size of the magnetite particles is around $12 \mathrm{~nm}$. The thickness of phospholipids shell is around $7 \mathrm{~nm}$, which coincides with the thickness of natural cell membranes. This result indicates that the shell of ML is phospholipid bilayer, which is absorbed directly onto the iron oxide core. The lauric acid was displaced with phospholipids due to the high affinity between the ferric ions and the phosphate group of the lipid. 
Ongoing work includes modification of the magnetoliposome with specific bio-molecules and complex core-shell alloy magnetic nanoparticles. The presentation will also cover cryo-section AEM and energy-filtered imaging for spatially-distributed chemical mapping.

[1] U. Hafeli et al eds. Proceed. 1st Intern. Conf. on Scientific and Clinical Applications of Magnetic Carriers. Rostock, Germany, Sep. 5-7, 1996. Plenum Press, NewYork, 1997.

[2] U. Hafeli et al eds. Proceed. 6th Intern. Conf. on Scientific and Clinical Applications of Magnetic Carriers, Tallahassee, Florida May 9-11, 2002. J. Magn. and Magn. Mater., 225 (2001).

[3] B.M. Lacava, et al. Appl. Phys. Lett. 77 (2000) 1876.

[4] L. Fu, V.P. Dravid, D.L. Johnson, Appl. Surf. Sci. 181 (2001) 173-178

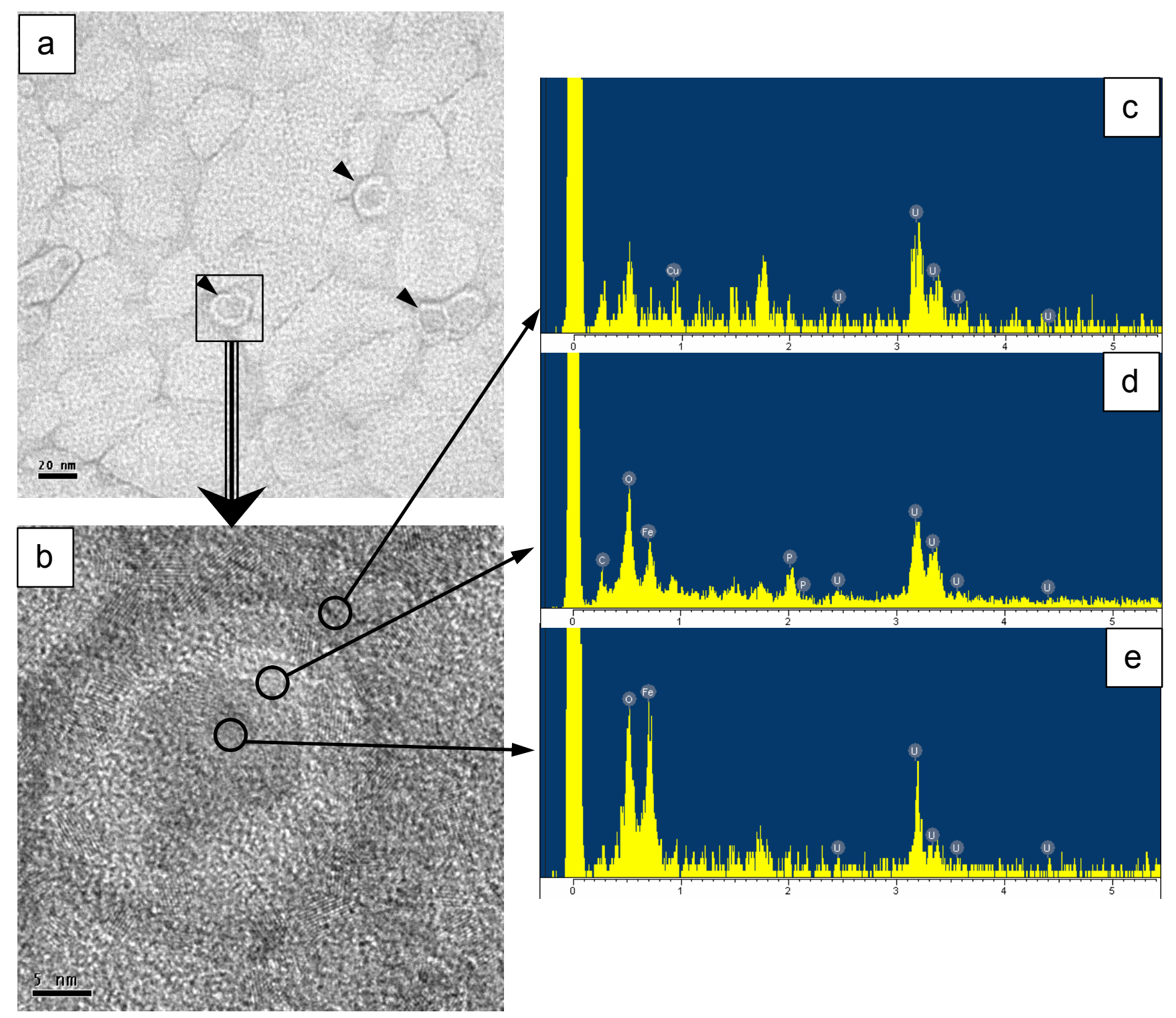

Figure 1. (a) TEM micrograph of magnetoliposome (ML), negatively stained with 5\% Uranyl Acetate. (b) Enlargement of one particle shown in (a). (c) EDX spectrum from outside of particle shows mainly Uranium peaks. (d) EDX spectrum from edge of the particle shows both $\mathrm{Fe}_{3} \mathrm{O}_{4}$ peaks and $\mathrm{U}$ paeks. (e) EDX spectrum from center of the particle shows major $\mathrm{Fe}_{3} \mathrm{O}_{4}$ peaks. 